\title{
Determinación de cómo el alumbrado de la Ciudad Universitaria afecta la calidad del cielo nocturno del OACS
}

Ricardo Antonio Pastrana Sánchez

\section{Resumen}

La contaminación lumínica es uno de los factores responsables de la atenuación del brillo de los cuerpos celestes, esto se debe principalmente a la forma inadecuada en que iluminamos nuestras ciudades. El objetivo que nos propusimos en este trabajo fue determinar en qué medida el alumbrado público instalado en la ciudad universitaria y sus alrededores afecta la observación astronómica del Observatorio Astronómico Centroamericano de Suyapa, y proponer las medidas para corregirlo. Los datos fueron obtenidos mediante el uso de luxómetro el cual se colocaba paralelo a la calle y a una altura de 1.5 metros, además mediante técnicas de GPS se georreferenciaron las zonas de estudio. Resultados encontrados en vías principales del campus universitario de la UNAH - Tegucigalpa están entre 3 lux y 95.5 lux que al ser comparados con estándares internacionales confirman la existencia de zonas en las cuales la iluminación es muy pobre o están sobre iluminadas. Nuestro trabajo consistió en el cálculo de la iluminancia media (Em), que se determinó mediante la aplicación del método estándar europeo de los "Nueve puntos".

Palabras clave: Contaminación lumínica, fotómetro, OACS, SQM, iluminancia, nueve puntos

\section{Abstract}

Light pollution is one of the factors responsible for the attenuation of the brightness of celestial bodies, this is mainly due to the inadequate way we light our cities. The goal we set in this work was to determine to what extent the public lighting installed in the campus and surrounding astronomical observation affects the Central Astronomical Observatory of Suyapa, and propose corrective action. The data were obtained by using LUXOMETRO which stood parallel to the street and a height of 1.5 meters, and using GPS techniques georeferenced studio areas. Results found in major ways UNAH college campus - Tegucigalpa are between 3 lux and 95.5 lux when compared to international standards confirm the existence of areas where the 
lighting is very poor or are over lit. Our work consisted in the calculation of the average illuminance $(\mathrm{Em})$, which was determined by applying the European standard method of the "Nine Points".

Keywords: Light pollution, photometer, OACS, SQM, illuminance, nine points.

Ricardo Antonio Pastrana Sánchez Departamento de Astronomía y Astrofísica, Facultad de Ciencias Espaciales, Universidad Nacional Autónoma de Honduras 


\section{Introducción}

Un cielo totalmente oscuro y limpio al momento de realizar una observación, es el deseo de todo astrónomo pero esto no suele suceder. La atmosfera de nuestro planeta se ve afectada por dos razones básicas: La primera se debe a la contaminación atmosférica causada por los gases que producen las industrias, fabricas, autos, etc. La segunda es la contaminación lumínica (CL) ocasionada por la manera inadecuada de iluminar nuestras ciudades.

Prácticas de iluminación concebidas de forma errónea son el uso masivo de luminarias, proyectores inclinados, letreros de publicidad luminosos que permanecen encendidos durante toda la noche emitiendo luz con intensidades excesiva (ver figura 1). Con esto se incrementa la $\mathrm{CL}$ haciendo que la luz de las estrellas, galaxias y demás astros se vea atenuada progresivamente.

Nuestra atmosfera dispersa la luz emitida por fuentes instaladas en zonas urbanas, creando un halo de luz visible sobre la ciudad aún desde gran distancia (Hosseini y Nasiri, 2007), al momento de la observación astronómica el telescopio capta tanto la luz del astro bajo estudio como la luz artificial que ha sido reflejada por la atmosfera.

La protección del cielo nocturno y la creciente disminución de nuestra capacidad de observar el universo ha sido motivo para que astrónomos y organizaciones mundiales suscribieran la DECLARACIÓN SOBRE LA DEFENSA DEL CIELO NOCTURNO Y EL DERECHO A LA LUZ DE LAS ESTRELLAS el 20 de abril de 2007 en La Palma, Islas Canarias, España.

El OACS no es ajeno a este problema ambiental, conocer el grado en que las luminarias cercanas afectan la observación astronómica contribuye a plantear alternativas que corrijan la iluminancia de la zona para el disfrute del cielo y la investigación astrofísica.

Al no contar con una normativa propia que regule la manera de iluminar los lugares destinados a la actividad humana, se hace necesaria la aplicación de métodos internacionales estándares para conocer la situación actual en esta área. El llamado método de los nueve puntos, especificado en la ITC-EA-07 del Reglamento de eficiencia energética del alumbrado exterior del gobierno de España fue el empleado en la captura de datos. 


\section{Equipo utilizado}

Para la determinación de la Em fue necesaria la medición de la iluminación producida por las luminarias del alumbrado público de la UNAH. El equipo empleado durante esta etapa de la investigación fue un luxómetro modelo CA811 la tabla 1 muestra las especificaciones técnicas del instrumento, un trípode con nivel, una cinta métrica de $20 \mathrm{~m}$, una plomada, GPS eTrex de Garmin las especificaciones aparecen en la tabla 2 y cámara fotográfica Cannon Rebel modelo eos350D.

El luxómetro transforma la energía luminosa en una señal eléctrica, la cual es medida en una escala calibrada. Este dispositivo es colocado en el trípode de modo que el sensor de silicio se mantenga paralelo a la superficie. Esto permite medir el flujo luminoso que incide sobre una superficie por unidad de área a la que llamamos iluminancia (E) y está dada por:

$$
E(\operatorname{lux})=\frac{d F}{d S}
$$

Donde $\mathrm{dF}$ es el diferencial del flujo luminoso expresado en lumen y dS el diferencial de superficie en $\mathrm{m}^{2}$. Nuestro medidor de luz fue ajustado para usar el lux (lumen $/ \mathrm{m}^{2}$ ) como unidad de medida.

\section{Metodología}

El OACS se encuentra inmerso en urbanizaciones que desde horas tempranas las luminarias del alumbrado público comienzan a iluminar (ver figura 2,) esto representa un grave problema para los astrónomos puesto que el cielo nocturno de la ciudad rápidamente pierde su calidad para la observación astronómica.

El brillo del cielo urbano está atenuando la luz proveniente de las estrellas y el universo. La contaminación lumínica es un efecto negativo que produce la forma indebida de la instalación de lámparas en vías públicas y el exceso de iluminación en ciertas aéreas.

Un cielo nocturno estrellado es un recurso de gran importancia para científicos y artistas así como para la sociedad y la cultura (Grigore, 2009). Las observaciones astronómicas se ven afectadas por el derroche energético que se hace en algunas zonas. Honduras no dispone de una normativa que indique el modo en que se debe iluminar un lugar específico. 
Para el cálculo de la Em fue necesaria la aplicación del método de los nueve puntos especificado en la ITC-EA-07 del Reglamento de Eficiencia Energética en instalaciones de alumbrado exterior del Gobierno de España. El método es estándar y posibilita la fácil obtención de datos. Este es el mismo método usado en los trabajos académicos Mapa de Contaminación Lumínica de la UCM (Universidad Complutense de Madrid) (Rodríguez, 2009) y Contaminación lumínica en la UCM (2010) I. Evolución de la iluminación en la UCM (Herranz, 2010).

El método consiste en tomar lecturas de iluminancia de un par de luminarias en 15 puntos contenidos en un rectángulo (ver figura 3). Los lados del paralelogramo lo forman la distancia entre las luminarias $(\mathrm{S})$ y la calzada de la calle (A). Los puntos son las intercepciones de las abscisas $B, C, D$, con las ordenadas 1, 2, 3,4 y 5 que se logran valiéndose de una cinta métrica. Cada zona de medición se ha georreferenciado usando técnicas GPS.

Las lecturas de iluminancia de cada punto se realizaron mediante la acopladura de trípode más luxómetro y una plomada (ver figura 4), la que permitía precisar el punto a medir. El sensor se fijaba paralelo a la calle a $1.50 \mathrm{~m}$ de altura.

El cálculo de Em viene dado por la ecuación:

$$
E m=\frac{E_{1}+2 E_{2}+E_{3}+2 E_{4}+4 E_{5}+2 E_{6}+E_{7}+2 E_{8}+E_{9}}{16}
$$

La medida real de la iluminancia en cada punto $\left(E_{i}\right)$ es la media aritmética de las medidas obtenidas en las abscisas. Por lo que $\mathrm{E}_{1}$ a $\mathrm{E}_{9}$ vienen dadas por:

$E_{1}=\left(B_{1}+B_{5}\right) / 2, E 2=\left(C_{1}+C_{5}\right) / 2, E_{3}=\left(D_{1}+D_{5}\right) / 2, E_{4}=\left(B_{2}+B_{4}\right) / 2, E_{5}=\left(C_{2}+C_{4}\right)$ /2, $E_{6}=\left(D_{2}+D_{4}\right) / 2, E_{7}=B_{3}, E_{8}=C_{3} y E_{9}=D_{3}$.

El valor calculado de Em también es usado para evaluar la uniformidad media (Um) de iluminancia, lo cual es el cociente entre el valor mínimo de las iluminancias Ei y la Em. Por otra parte la determinación de la uniformidad general (Ug) se calcula dividiendo el valor mínimo de las iluminancias Ei entre el valor máximo de dichas iluminancias. 


\section{Resultados}

Se ha cubierto un área total de $3,334.1 \mathrm{~m}^{2}$. El cálculo para el valor mínimo y máximo de la Em fue de 1 y 219 lux respectivamente. Los valores encontrados están fuera de los rangos establecidos para la iluminación del alumbrado público del ayuntamiento de Madrid.

Los datos encontrados que se resumen en la tabla 3 revelan que las zonas bajo estudio presentan valores de iluminación muy pobres y en otras ocasiones están sobre iluminadas. El cociente de Um indica que los valores aceptados deben de estar dentro de un rango de 0.2 a 0.5 . Nuestros cálculos reflejan valores que van desde 0 a 1.36 determinando que no se ha encontrado zona alguna que este en el parámetro aceptado internacionalmente.

Las magnitudes estándar de Em, Um y Ug para cada zona de estudio se especifican en la tabla 4. La Ug nos indica que tan pareja se encuentra la iluminación de las zonas estudiadas. Esta toma en cuenta todos los puntos de la calzada donde se encuentra los valores mínimos, máximos y promedios de luminancia, salvo en pocos casos como las mediciones 5,10,14,16 y 18 los resultados calculados se encuentran dentro de los valores estándares.

\section{Conclusiones}

Los coeficientes calculados de la Ug, advierten que la mayor parte de las zonas investigadas indican que la radiación que incide sobre ellas no está distribuida de forma pareja.

La disparidad de las magnitudes encontradas de Em indica que la UNAH no cuenta con una eficiente iluminación de sus áreas destinadas al que hacer humano. Causando en algunos casos excesos de radiación innecesarios y en otros falta de luz artificial.

La estadística registrada en el presente trabajo contribuirá a realizar una caracterización más completa de la Em, Um y Eg del campus universitario y sus efectos sobre el OACS.

La investigación realizada permitirá realizar estudios futuros de la evolución del alumbrado público de la UNAH, detectando correcciones, excesos o escaza iluminación pública. 


\section{Recomendaciones} siguiente.

A fin de mejorar las condiciones de observación en el OACS se sugiere lo

1. Se deben realizar los cambios de las luminarias que se detectaron el mal estado.

2. Es de suma importancia que se haga un trabajo de poda de árboles que bloquean la luz emitida por el alumbrado público con el propósito de mejorar la iluminación a nivel de suelo.

3. Se debe realizar los ajustes de energización de las luminarias para que estas emitan dentro de los rangos establecidos internacionalmente.

4. Realizar mediciones del Em en futuras fechas permitirá conocer si la iluminación pública de la UNAH se mantiene bajo control.

5. Establecer un reglamento interno de la UNAH sobre el alumbrado público. A la vez este serviría como modelo para las autoridades del gobierno tomen conciencia de la importancia de iluminar correctamente y ahorro que representa en la factura energética nacional.

\section{Bibliografía}

- Grigore, V. (2009). Meteor Observation and the Light Pollution. Proceedings of the International Meteor Conference, 68 - 75.

- Herranz, I. R. (2010). Contaminación lumínica en la UCM (2010) I. Evolución de la iluminación en la UCM. Madrid, España: Departamento de Astrofísica y CC. de la Atmósfera.

- MINISTERIO DE INDUSTRIA, T. Y. (Miércoles 19 de Noviembre de 2008). REAL DECRETO 1890/2008. REGLAMENTO DE EFICIENCIA ENERGÉTICA EN INSTALACIONES DE ALUMBRADO EXTERIOR. Madrid, Madrid, España: Boletín Oficial del Estado núm. 279.

- Nasiri, S. H. (2007). Measurement of Light Pollution for Observatory Sites. Solar and Stellar Physics Through Eclipses, 284 - 288. 
- Rodríguez, P. C. (2009). Mapa de contaminación lumínica de la UCM lumínica de la UCM. Madrid, España: Departamento de Astrofísica y CC. de la Atmósfera.

- StarLight. (2007). DECLARACIÓN SOBRE LA DEFENSA DEL CIELO NOCTURNO Y EL DERECHO A LA LUZ DE LAS ESTRELLAS. Conferencia Internacional en Defensa de la Calidad del Cielo Nocturno y el Derecho a Observar las Estrellas (págs. 1 - 14). La Palma, Islas Canarias, España : @ Bob Crelin.

\section{Anexos:}

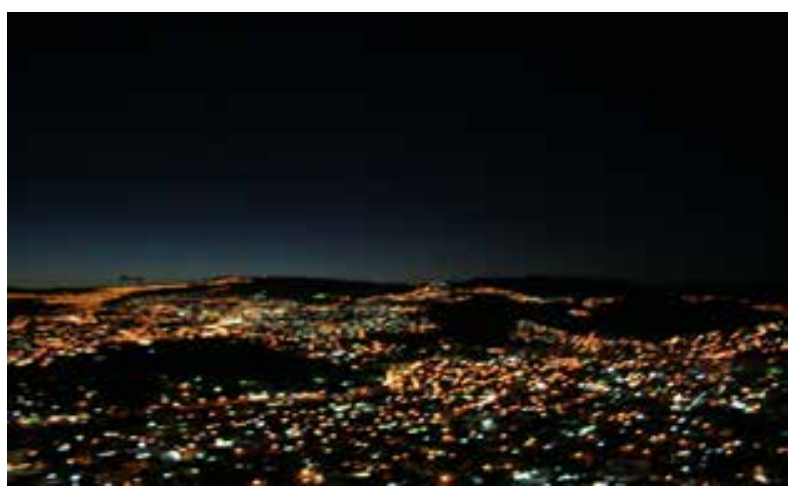

Figura 1. Toma nocturna de Tegucigalpa.

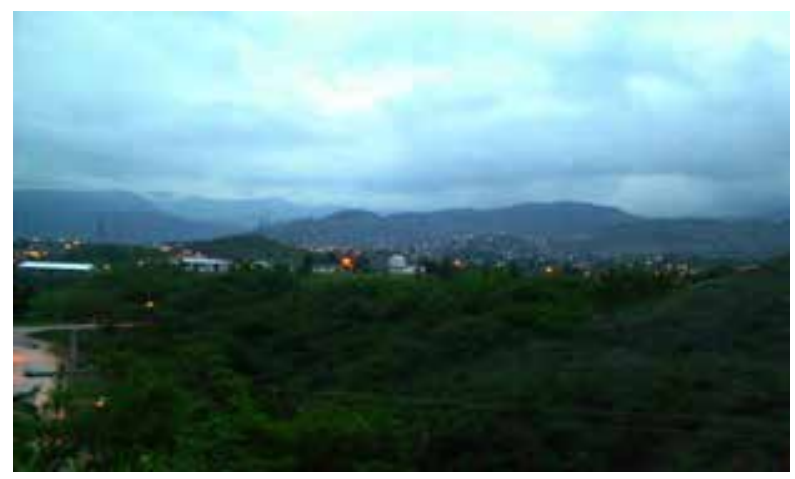

Figura 2. Fotografía del OACS al fondo puede verse las luminarias encendidas aún cuando el Sol se encuentra sobre el horizonte. 


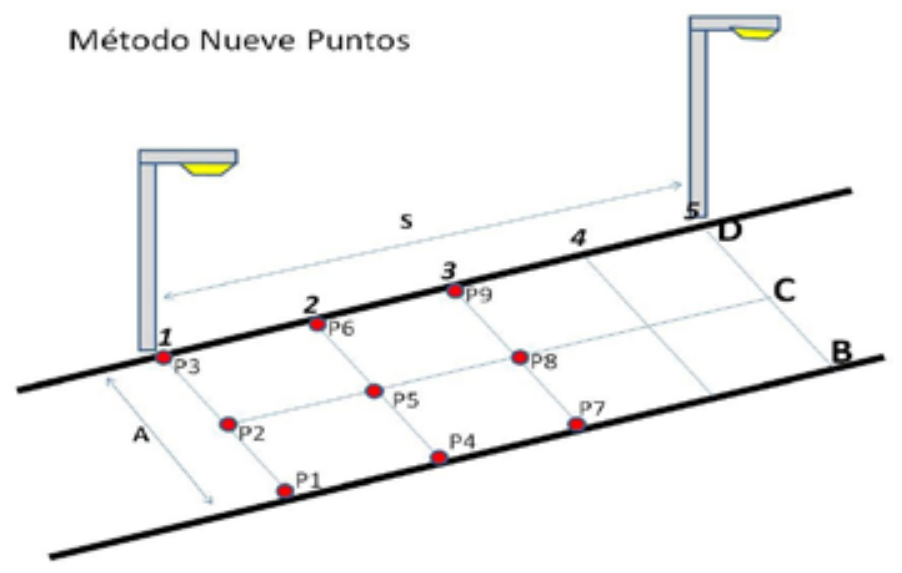

Figura 3. Método estándar de los nueve puntos para el cálculo de la iluminancia media.

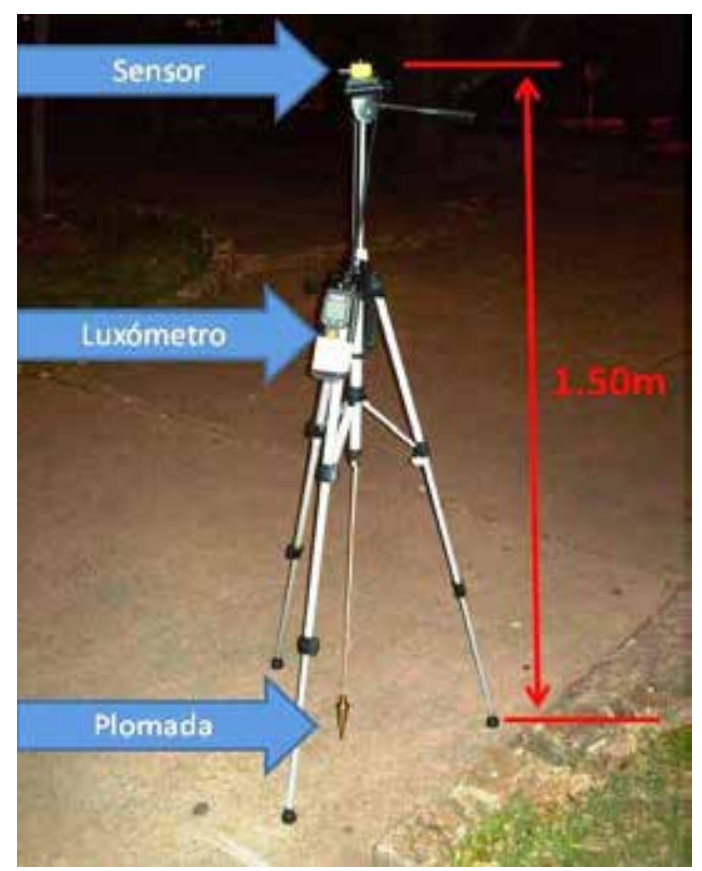

Figura 4. Equipo utilizado durante las mediciones de iluminación. 
Tabla 1. Características técnicas del luxómetro.

\begin{tabular}{|c|c|}
\hline \multicolumn{2}{|c|}{ Especificaciones luxómetro CA811 } \\
\hline Magnitud & Valor \\
\hline \multirow{2}{*}{ Rango } & 20fc, 200fc, 2000fc, 20kfc \\
\cline { 2 - 2 } & 20lux, 200lux, 2000lux, 20klux \\
\hline Resolución & $0.1 \mathrm{fc} 0$ 0.1lux \\
\hline Sensor & Fotodiodo de Silicio \\
\hline Respuesta spectral & Curva fotóptica CIE ${ }^{*}$ \\
\hline Precisión para una fuente de luz de 2856k & $\pm 3 \% \pm 10$ dig \\
\hline
\end{tabular}

${ }^{*} \mathrm{CIE}$ : siglas en francés de la Comisión Internacional de lluminación.

Tabla 2. . Características técnicas del GPS.

\begin{tabular}{|c|c|}
\hline \multicolumn{2}{|c|}{ Especificaciones GPS eTrex } \\
\hline Magnitud & Medida \\
\hline Receptor & Compatible con WAAS* \\
\hline \multirow{3}{*}{ Tiempo de adquisición } & $\sim 3 \mathrm{seg}$ (caliente) \\
\cline { 2 - 2 } & $\sim 32 \mathrm{seg}$ (tibio) \\
\cline { 2 - 2 } $\begin{array}{c}\text { Frecuencia de actualiza- } \\
\text { ción }\end{array}$ & $\sim 39 \mathrm{seg}$ (frio) \\
\hline Precisión GPS & $<$ segundo, continua \\
\hline \multirow{2}{*}{$\begin{array}{c}\text { Presición DGPS } \\
\text { (WAAS) }\end{array}$} & 3 metros (10 pies) \\
\hline Presición de velocidad & 0.1 nudos RMS en estado estable \\
\hline
\end{tabular}

* WAAS (Wide Area Augmentation System), Sistema de corrección diferencial satelital.

** DGPS (Differential GPS), Sistema que proporciona a los GPS correcciones de datos. 


\begin{tabular}{||c|c|c|c|}
\hline Lugar (Madrid) & Iluminancia (lux) min/max & Lugar (UNAH) & $\begin{array}{c}\text { Iluminancia (lux) } \\
\text { min/max }\end{array}$ \\
\hline Pase0s & $7 / 10$ & Paseo A1-Fisica & $5.65 / 12.78$ \\
Pase0s & $7 / 10$ & Paseo Rectoria - A1 & $10 / 219$ \\
\hline $\begin{array}{c}\text { Pase0 } \\
\text { superficie }\end{array}$ & $7 / 10$ & Paseo D1 & $1 / 17.5$ \\
\hline \begin{tabular}{c} 
Vlas Principales \\
\hline
\end{tabular} & $15 / 20$ & Estacionamiento Padre Trino & $1 / 127$ \\
\hline
\end{tabular}

Tabla 3. Resumen de los valores encontrados durante las mediciones. 


\begin{tabular}{||c|l|c|c|c|}
\hline \multirow{2}{*}{ Zona } & No. De Medición & Iluminancia media & Uniformidad media & Uniformidad general \\
& Em & Um & Ug \\
Paseo Edificio & Medición 1 & 12.1875 & 1.066666667 & 0.472727273 \\
A1 y Fisica & Medición 2 & 12.71875 & 0.157248157 & 0.066666667 \\
\cline { 2 - 5 } & Medición 3 & 8.1875 & 0.610687023 & 0.4 \\
Paseo Rectoría y & Medición 4 & 5.71875 & 0 & 0.148148148 \\
A1 & Medición 5 & 43.28125 & 0.323465704 & 0.282828283 \\
Paseo principal & Medición 6 & 4.40625 & 1.361702128 & 2 \\
D1 & Medición 7 & 8.0625 & 0.744186047 & 0.923076923 \\
& Medición 8 & 34.9375 & 1.14490161 & 1.25 \\
& Medición 9 & 41.5 & 1.34939759 & 1.230769231 \\
Estacionamiento & Medición 10 & 11.1875 & 0.357541899 & 0.097560976 \\
\cline { 2 - 5 } Padre Trino & Medición 11 & 48.0625 & 1.061118336 & 1 \\
\cline { 2 - 5 } & Medición 12 & 21.75 & 0.091954023 & 0.078431373 \\
& Medición 13 & 37.625 & 1.063122924 & 0.85106383 \\
& Medición 14 & 22.53125 & 0.53259362 & 0.406779661 \\
& Medición 15 & 55.28125 & 1.26625212 & 1.147540984 \\
& Medición 16 & 14.3125 & 0.349344978 & 0.208333333 \\
Piscina & Medición 17 & 16.8125 & 0.594795539 & 0.357142857 \\
& Medición 18 & 16.71875 & 0.358878505 & 0.181818182 \\
\hline \hline
\end{tabular}

Tabla 4. Valores de lluminancia media, Uniformidad media y Uniformidad general. 